\title{
MODELLING OF AUTOGENOUS HEALING FOR REGULAR CONCRETE VIA A DISCRETE MODEL
}

\author{
A. CIBELLI* , G. DI LUZIO*, L. FERRARA*, G. CUSATIS ${ }^{\dagger}$, M. PATHIRAGE ${ }^{\dagger}$ \\ *Politecnico di Milano \\ Department of Civil and Environmental Engineering (DICA) \\ Piazza Leonardo da Vinci 32, 20133 Milan, Italy \\ e-mail: antonio.cibelli@polimi.it, giovanni.diluzio@polimi.it, liberato.ferrara@polimi.it \\ ${ }^{\dagger}$ Northwestern University \\ Department of Civil and Environmental Engineering \\ McCormick School of Engineering and Applied Science \\ Evanston, IL 60208 USA \\ e-mail: g-cusatis@northwestern.edu,madurapathirage2014@u.northwestern.edu
}

Key words: Autogenous healing, Self-healing model, Regular concrete, Hygro-Thermo-Chemical model, Lattice Discrete Particle Model, Strength recovery, Numerical modelling

\begin{abstract}
In this paper a numerical model for autogenous healing of normal strength concrete is presented in detail, along with preliminary results of its validation, which is planned to be achieved by comparing the results of numerical analyses with those of a dedicated experimental campaign. Recently the SMM (Solidification-Microprestress-Microplane model M4) model for concrete, which makes use of a modified microplane model M4 and the solidification-microprestress theory, has been extended to incorporate the autogenous healing effects. The moisture and heat fields, as well as the hydration degree, are obtained from the solution of a hygro-thermo-chemical problem, which is coupled with the SMM model. The updated model can also simulate the effects of cracking on the permeability and the restoring effect of the self-healing on the mechanical constitutive laws, i.e. the microplane model. In this work, the same approach is introduced into a discrete model, namely the Lattice Discrete Particle Model (LDPM). A numerical example is presented to validate the proposed computational model employing experimental data from a recent test series undertaken at Politecnico di Milano.
\end{abstract}

\section{INTRODUCTION}

Nowadays durability of concrete is a topic of utmost relevance because of several different yet complementary reasons. The willing of building longer lasting structures in order for concrete to result in a less environmentaffecting material, the need for developing sound quantitative models capable of predicting the structural life span of concrete constructions, and the necessity to design such structures by means of approaches which would ac- count for durability as an actual performance, which the technician can predict, assess and take action on, design-wise, are only three of the many concurrent causes that have made this topic worth deserving an increasing interest by the scientific community.

Material-speaking, a Ultra High Durability Concrete (UHDC) is what would make concrete structures last more, even in Extremely Aggressive Exposures (EAE). Therefore, such a material might be the answer for both sustainabil- 
ity and less maintenance demanding constructions which the construction sector itself seems to ask for today. On the other side, a Durability Assessment-based Design is also necessary, since in current design approaches, lifespan is defined by a target reference value, which, through a "structural class concept" and as a function of the exposure class, results into prescriptions deemed to guarantee the demanded level of durability. It is worth remarking that, generally speaking, all the models, so far developed and also implemented into the design codes, do not yet consider the cracked state of the concrete [1]. This represents a huge limitation, since concrete elements undergo cracking in service conditions, and precisely these cracks represent the way in for aggressive agents to activate material degradation processes.

Both laboratory experimental tests and monitoring campaigns on large scale proof-of-concepts of UHDC in aggressive exposures, together with the development and validation of numerical models as basis of a qualitative approach to predict long-term performances of concrete constructions are all objectives of the H2020 project ReSHEALience (GA 780624).

This paper is the first result of a three-year $\mathrm{Ph} . \mathrm{D}$. program, which aims to move some hopefully meaningful steps towards the computational modelling of long-term performances and degradation of concrete and reinforced concrete structures. In particular, the present work is focused on the numerical modelling of autogenous healing of a regular concrete. In the subsequent section a general and brief presentation of two numerical models clarify the background from where this study moves: the Hygro-Thermo-Chemical (HTC) model and the Lattice Discrete Particle Model (LDPM). The former dealing with the diffusion part of the problem, whereas the latter is the mechanical model. Then, the self-healing phenomenon for concrete is concisely presented, just before moving to how it is implemented into the numerical framework directly deriving from the theoretical models above mentioned. In the last two sections experimental tests and numerical analyses are described, and finally results are discussed and compared. To conclude, some remarks relevant to the authors are reported, trying to underline the most meaningful outcomes and limitations of the still ongoing research work.

\section{RESEARCH BACKGROUND}

Recently the SMM (SolidificationMicroprestress-Microplane model M4) model for concrete, which makes use of a modified microplane model M4 [2] and the solidificationmicroprestress theory, has been extended to incorporate the self-healing effects and, in particular, the effect of delayed cement hydration, which is the main cause of the self-healing for young concrete [3]. The moisture and heat fields, as well as the hydration degree, have been obtained from the solution of a hygrothermo-chemical problem, which has been coupled with the SMM model. Such a model can also simulate the effects of cracking on the permeability and the restoring effect of the selfhealing on the mechanical constitutive laws, i.e. the microplane model.

In this paper, the approach presented above is introduced into a discrete model, namely the Lattice Discrete Particle Model (LDPM). For the sake of clarity, the main features of both theoretical models are briefly outlined in the next two subsections. All the details of these formulations, the theoretical part as well as the numerical side, can be found in Refs. [4-7].

\subsection{Hygro-Thermo-Chemical model}

To exhaustively model the behaviour of concrete, from early age onwards, two crucial parameters must be paid attention to: relative humidity, $h$, and temperature, $T$, whose spatial distribution over the domain together with their evolution in time significantly affect the overall response of concrete to both mechanical and environmental boundary conditions. Once the analysis domain has been identified, temperature and relative humidity fields can be evaluated by enforcing the enthalpy (2) and moisture content (1) mass balances, respectively. 


$$
\begin{gathered}
\nabla \cdot\left(D_{h} \nabla h\right)-\frac{\partial w_{e}}{\partial h} \cdot \frac{\partial h}{\partial t}+ \\
-\left(\frac{\partial w_{e}}{\partial \alpha_{c}} \dot{\alpha}_{c}+\frac{\partial w_{e}}{\partial \alpha_{s}} \dot{\alpha}_{s}+\dot{w}_{n}\right)=0 \\
\nabla \cdot\left(\lambda_{t} \nabla T\right)-\rho c_{t} \frac{\partial T}{\partial t}+ \\
\left(\dot{\alpha}_{c} c \tilde{Q}_{c}^{\infty}+\dot{\alpha}_{s} s \tilde{Q}_{s}^{\infty}\right)=0
\end{gathered}
$$

where $D_{h}(h, T)$ is a proportionality coefficient, non-linear function of $h$ and $T$ and called moisture permeability, $\lambda_{t}$ is the heat conductivity, $\alpha_{c}$ is the cement hydration degree, $\alpha_{s}$ is the "supplementary cementitious material" reaction degree, $w_{e}$ and $w_{n}$ represent the evaporable and non-evaporable (chemically bound) fractions of the water content $w, c_{t}$ is the isobaric heat capacity (specific heat), $c$ is the cement content, and $\tilde{Q}_{c}^{\infty}$ is the latent heat of cement reaction per unit mass of reacted cement.

The resulting system of two PDEs (1/2) properly describes the two-field problem by means of two state variables, namely $h$ and $T$; in addition, several internal parameters are used in order to enrich the diffusion problem in regard to the chemical reactions taking place within the material.

As it clearly arises by looking at equations (1) and (2), both phenomena, moisture diffusion and heat transfer, are strongly affected by cement hydration [4, 8,-13], supplementary cementitious material reactions [4] and silicate polymerisation [4]: all these three processes are deeply analysed in [4, 5], where all the details about how they are modelled, calibrated and validated are provided.

In the HTC model, the strength evolution is assumed to depend on the so-called ageing degree, $\lambda$, defined as

$$
\dot{\lambda}=\left(\frac{T_{\max }-T}{T_{\max }-T_{\text {ref }}}\right)^{n_{\lambda}}\left(B_{\lambda}-2 A_{\lambda} \alpha\right) \dot{\alpha}
$$

for $\alpha>\alpha_{0}$ and $\dot{\lambda}=0$ otherwise. The parameter $\alpha_{0}$ defines the value of the reaction degree at the end of the setting phase. Values of $\alpha_{0}=0.1 \div 0.4$ have been proposed in the literature, depending on type of cement and water-to-cement ratio. $T_{\max }$ represents the maximum temperature at which hardening of concrete is possible under standard conditions $\left(\approx 100^{\circ} \mathrm{C}\right) . T_{r e f}$ is the reference temperature for the experimental calibration of the ageing model, thus for $T=T_{\text {ref }}$, it must result $\lambda=0$ for $\alpha \leq \alpha_{0}$ and $\lambda=1$ for $\alpha=\alpha_{\infty}$. By imposing these conditions one obtains $B_{\lambda}=$ $\left[1+A_{\lambda}\left(\alpha_{\infty}^{2}-\alpha_{0}^{2}\right)\right] /\left(\alpha_{\infty}-\alpha_{0}\right)$, in which $n_{\lambda}$ and $A_{\lambda}$ are two model parameters that need to be identified from experimental data.

\subsection{Lattice Discrete Particle Model}

At the meso-scale concrete can be considered as a two-phase material, consisting of mortar and coarse aggregates. LDPM simulates concrete mesostructure by considering only the coarse aggregate particles, typically larger than $4 \mathrm{~mm}$, in order to have reasonable computational burden. The mesostructure in LDPM is defined through the following steps. 1) Particles are generated accordingly to a particle size distribution function, in turn, associated to a sieve curve conceived in a Fuller fashion (Figs. 1a,b). 2) The coarse aggregate particles, whose shape is assumed to be spherical, are introduced into the concrete volume by a try-and-reject random procedure (Fig. 1c). 2) Zero-radius aggregate particles (nodes) are randomly distributed over the external surfaces. 3) A three-dimensional domain tessellation (Fig. 1d), based on the Delaunay tetrahedralization of the generated aggregate centres, creates a system of cells interacting through triangular facets (Fig. 1e).

Vectorial constitutive laws governing the behaviour of the model are imposed at the centroid of the projection of each single facet (contact point) onto a plane orthogonal to the straight line connecting the particle centres (edges of the tetrahedralization). The projections are used instead of the facets to ensure that the shear interaction between adjacent particles does not depend on the shear orientation.

The straight lines connecting the particle centres define the lattice system (Figs. 1f-h).

Rigid body kinematics describes the displace- 
ment field along the lattice struts and the displacement jump, $\llbracket \mathbf{u}_{C} \rrbracket$, at the contact point (Fig. 1i). The strain vector is defined as the displacement jump at the contact point divided by the inter-particle distance, $L$. The components of the strain vector in a local system of reference, characterised by the unit vectors $\mathbf{n}, \mathbf{l}$ and $\mathbf{m}$, are the normal and shear strains. The unit vector $\mathbf{n}$ is orthogonal to the projected facet and the unit vectors $\mathbf{I}$ and $\mathbf{m}$ are mutually orthogonal and lie on the projected facet. Concerning the elastic behaviour, it is described by assuming that the normal and shear stresses are proportional to the corresponding strains. Then, the elastic constitutive laws along the three local directions read:

$$
\begin{array}{rlr}
\varepsilon_{N} & =\frac{\mathbf{n}^{T} \llbracket \mathbf{u}_{C} \rrbracket}{L} ; \quad \sigma_{N}=E_{N} \varepsilon_{N} \\
\varepsilon_{L} & =\frac{\mathbf{l}^{T} \llbracket \mathbf{u}_{C} \rrbracket}{L} ; & \sigma_{L}=E_{T} \varepsilon_{L} \\
\varepsilon_{M}=\frac{\mathbf{m}^{T} \llbracket \mathbf{u}_{C} \rrbracket}{L} ; & \sigma_{M}=E_{T} \varepsilon_{M}
\end{array}
$$

where $E_{N}=E_{0}, E_{T}=\alpha E_{0}, E_{0}$ is the effective normal modulus, and $\alpha$ is the shearnormal coupling parameter. $E_{0}$ and $\alpha$ are assumed to be material properties. At the macroscopic level, concrete elastic behaviour is statistically homogeneous and isotropic. As such, it can be modelled effectively through the classical theory of elasticity characterized by Young's modulus, $E$, and Poisson's ratio, $\nu$. The relationship between the meso-scale LDPM parameters, $E_{0}$ and $\alpha$, and the macroscopic elastic parameters, $E$ and $\nu$, can be obtained by considering a limiting case in which an infinite number of facets surrounds one aggregate piece. In this case, the LDPM formulation corresponds to the kinematically constrained formulation of the microplane model without deviatoric/volumetric split of the normal strain component and one can write

$$
E_{0}=\frac{1}{1-2 \nu} E \quad \text { or } \quad E=\frac{2+3 \alpha}{4+\alpha} E_{0}
$$

and

$$
\alpha=\frac{1-4 \nu}{1+\nu} \quad \text { or } \quad \nu=\frac{1-\alpha}{4+\alpha}
$$

The LDPM formulation of the nonlinear and inelastic behaviour aims at representing three separate physical mechanisms characterizing meso-scale failure behaviour: (1) fracturing and cohesive behaviour under tension and tension/shear; (2) pore collapse and material compaction under high compressive stresses; and (3) frictional behaviour. In this study, the numerical modelling of the self-healing induced effect is of interest; since the afore-mentioned effect plays a relevant role in strength recovery with regard to fracturing behaviour, the associate constitutive relationships are presented hereafter. Further details about the mechanical model, such as mesh features, discrete compatibility and equilibrium equations as well as a complete discussion about the constitutive laws can be found in the Refs. [6,7].

Fracturing and cohesive behaviour under tension and tension/shear.

It is convenient to formulate the fracture and damage evolution by a relationship between the effective strain, $\varepsilon$, and the effective stress, $\sigma$, defined as

$$
\begin{array}{r}
\varepsilon=\sqrt{\varepsilon_{N}^{2}+\alpha\left(\varepsilon_{L}^{2}+\varepsilon_{M}^{2}\right)} \\
\sigma=\sqrt{\sigma_{N}^{2}+\left(\sigma_{L}^{2}+\sigma_{M}^{2}\right) / \alpha}
\end{array}
$$

Thanks to the definition of the effective stress and strain, the relationship between normal and shear stresses, $\sigma_{N}, \sigma_{L}$, and $\sigma_{M}$, and associated strains, $\varepsilon_{N}, \varepsilon_{L}$, and $\varepsilon_{M}$, can be written through damage-type constitutive equations:

$$
\sigma_{N}=\sigma \frac{\varepsilon_{N}}{\varepsilon} ; \sigma_{L}=\sigma \frac{\alpha \cdot \varepsilon_{L}}{\varepsilon} ; \sigma_{M}=\sigma \frac{\alpha \cdot \varepsilon_{M}}{\varepsilon}
$$

The strain-dependent boundary $\sigma_{b t}(\varepsilon, \omega)$ can be expressed as

$$
\sigma_{b t}=\sigma_{0} \cdot \exp \left[-H_{0}(\omega) \frac{\left\langle\varepsilon_{\max }-\varepsilon_{0}(\omega)\right\rangle}{\sigma_{0}}\right]
$$

in which the brackets $\langle\cdot\rangle$ are used in Macaulay sense: $\langle x\rangle=\max \langle x, 0\rangle$.

In $(12), \omega=\arctan \left(\varepsilon_{N} / \sqrt{\alpha} \varepsilon_{T}\right)$ represents the coupling variable expressing the degree of interaction between shear and normal loading. Then, $\sigma_{0}$ is the strength limit for effective stress, in 

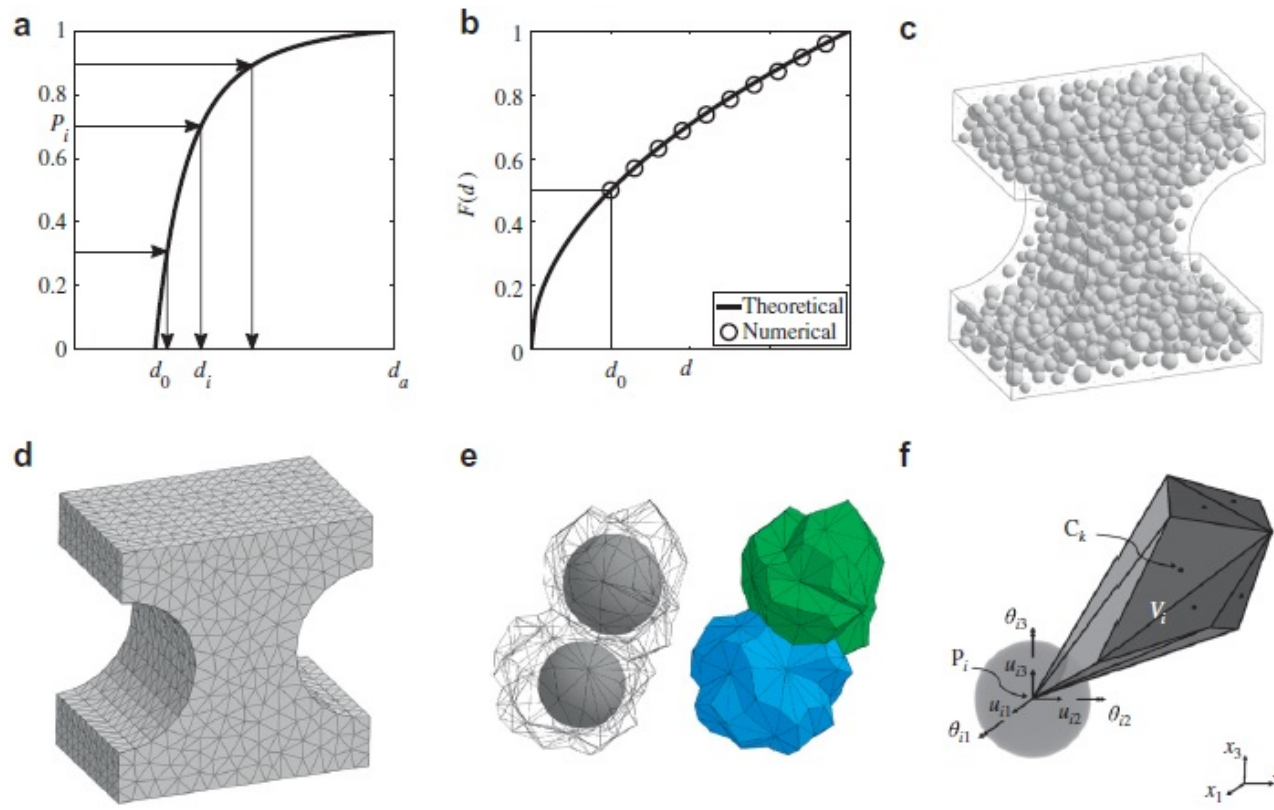

e

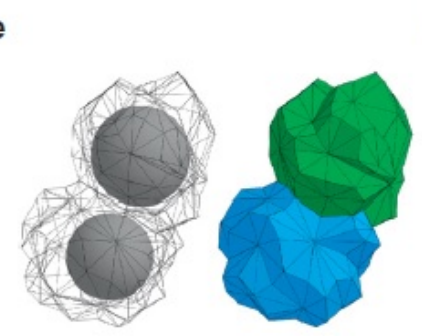

f

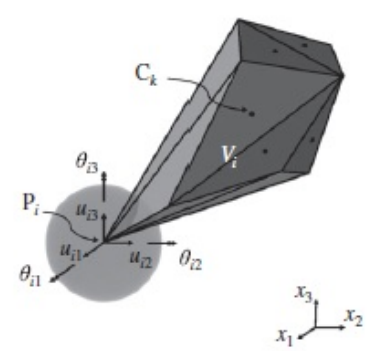

g

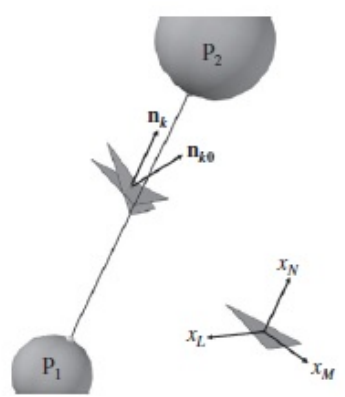

h
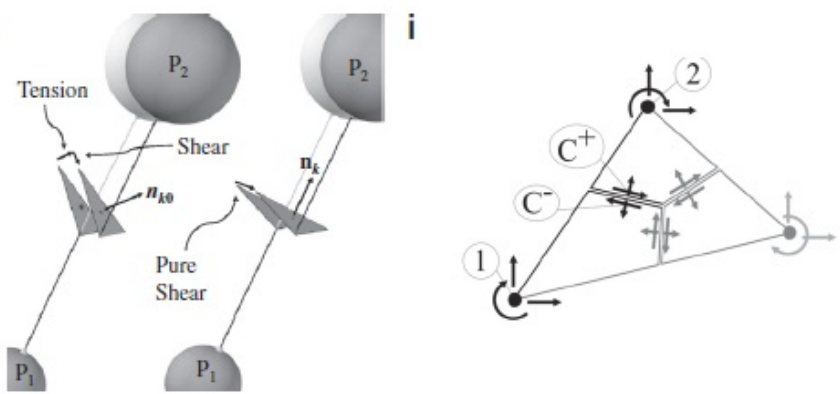

Figure 1: (a) Probability distribution function for particle size generation;(b) theoretical (solid curve) and numerical (circles) sieve curve; (c) particle system for a typical dog-bone specimen; (d) tetrahedralisation for a typical dog-bone specimen; (e) tessellation of a typical LDPM tetrahedron connecting four adjacent particles; (f) portion of a tetrahedron associated to one particle; (g) original and projected LDPM facets; (h) effect of meso-scale pure shear loading on one LDPM original facet (left) and one LDPM projected facet (right) and (i) definition of nodal degrees of freedom and contact facets in two-dimensions. [6]

turn function of $\omega$ and $r_{s t}$, which is the ratio between the shear strength (cohesion), $\sigma_{s}$, and the tensile strength, $\sigma_{t}$. Finally, $H_{0}$ is the socalled softening modulus: the parameter governing the exponential decay of the boundary $\sigma_{b t}$ after effective strain has reached its elastic limit, $\varepsilon_{0}=\sigma_{0}(\omega) / E_{0}[6]$.

\section{NUMERICAL IMPLEMENTATION OF AUTOGENOUS HEALING}

Concrete has the inherent capability of sealing and/or healing the cracks, depending on whether the recovery of mechanical properties occurs or not, after damage has been restored [16]. The chance of concrete repairing with no human actions, in recent times, has gathered the increasing interest of concrete professionals and scientific community; in fact, flourishing research and development activity has led to the concept and validation of countless techniques to promote and enhance the self-healing capacity of cement-based materials.

Concerning the differences standing between autogenous and engineered healing, it is worth mentioning the different achievable levels in terms of mechanical properties regain. When 
autogenic healing occurs, though the materials filling the cracks (secondary hydration products and $\mathrm{CaCO}_{3}$ crystals) are identical to the constituting elements of the cementitious matrix, the Calcium-Silicate-Hydrates (C-S-H) gel formed due to ongoing hydration seems to have lower mechanical properties compared to primary hydration products, and deposited crystals do not form a proper bond with the crack faces. This means that, for autogenic healing, mechanical properties of the pristine material will never be recovered completely. On the other hand, in case of engineered healing the complete regain in strength and stiffness can be obtained or even a performance higher than that of the virgin material.

Recently, an increasing interest is being addressing by the scientific community as well as the concrete professionals to the numerical modelling of healing phenomena [22], whether autogenous or "engineered". This study is intended to follow this trend, in particular it is focused on the autogenous healing, also called self-healing to emphasise the inherent nature of the phenomenon.

\subsection{Self-healing law}

The driving force of the overall healing process is the reaction affinity $A_{s h}\left(\lambda_{s h}\right)$ that can reasonably be assumed to decrease progressively while healing develops. At the beginning, $A_{s h}\left(\lambda_{s h}=0\right)=A_{s h 0}$, a condition making the reaction start and advance up to an equilibrium state, in which $A_{s h}\left(\lambda_{s h}=1\right)=0$ : as soon as this condition is reached, the healing process ceases. Assuming the affinity to vary linearly, it reads

$$
A_{s h}\left(\lambda_{s h}\right)=A_{s h 0}\left(1-\lambda_{s h}\right)
$$

Additionally, also the reaction rate $\dot{\lambda}_{s h}$ is supposed to be a linear function of the reaction affinity

$$
A_{s h}\left(\lambda_{s h}\right)=k_{s h} \dot{\lambda}_{s h}
$$

where $k_{s h}$ is a positive kinetic coefficient. By substituting $A_{s h}\left(\lambda_{s h}\right)$ in (13), one obtains a first order reaction kinetic law [17]

$$
\dot{\lambda}_{s h}=\tilde{A}_{s h}\left(\lambda_{s h}\right)\left(1-\lambda_{s h}\right)
$$

where $\tilde{A}_{s h}\left(\lambda_{s h}\right)=A_{s h 0} / k_{s h}$ represents the inverse of the characteristic time of the overall self-healing reaction. To set up a reasonable and physically sound expression for the initial reaction affinity $A_{s h 0}$ and, consequently, for the coefficient $\tilde{A}_{s h}$, it has to be considered that this coefficient depends on several different factors. (1) The extent of self-healing in cracked concrete was found to be highly dependent on the crack width, with smaller cracks healing generally better and at a faster rate than larger cracks. (2) The continued hydration is the principal cause of recovery. (3) The other fundamental process generating the self-healing is the calcium carbonate precipitation which is a rather straightforward chemical process governed mainly by four key factors: the calcium concentration, the concentration of dissolved inorganic carbon, the $\mathrm{pH}$, and the availability of nucleation sites [18, 19]. (4) The moisture supply is hence the most important factor for selfhealing, together with the age of the first cracking. Therefore, from those evidences in the literature, the quantities of unhydrated cement at the time of first crack and the moisture content in the pores of concrete near the crack are essential parameters for the autogenous healing process. According to the evidences recalled above the coefficient $\tilde{A}_{s h}$ can be expressed as

$$
\tilde{A}_{s h}=\tilde{A}_{s h 0} f_{h}(h) f_{w}\left(w_{c}\right) e^{-E_{s h} / R T}
$$

The fourth term in $(16)$, expressing the effect of current temperature, $T$, on the reaction rate, is of the Arrhenius type, as usual for thermally activated chemical reactions where $E_{s h}$ is the selfhealing activation energy and $R$ is the universal gas constant. The coefficient $\tilde{A}_{s h 0}$ accounts for the effect of the initial unhydrated cement and the possible presence of additive material that accelerates the healing process

$$
\tilde{A}_{s h 0}=\tilde{A}_{s h 1}\left(1-\alpha_{c}^{s h 0}\right) c+\tilde{A}_{s h 2} a d
$$

where $\alpha_{c}^{s h 0}$ is the value of the hydration degree when the healing process starts (that isn't 
generally uniform in the volume), $c$ is the cement content, so the product $\left(1-\alpha_{c}^{s h 0}\right) c$ represents the amount of unhydrated cement when the healing action begins, $a d$ is the content of healing boosting additive, and $\tilde{A}_{s h 1}$ and $\tilde{A}_{s h 1}$ are two material parameters that have the units of volume/mass/time. The first term weights the effect of all the chemical processes as described above. Here the effect of any supplementary "healing engineering" admixture is considered to be additive to the effect of the unhydrated cement because of the type of additive used in the experimental campaign. Different kind of additives may give different contribution to the affinity.

It is well known from experiments the importance of water or high level of relative humidity to activate the self-healing activity, which, below a certain value, decreases and may be even zero. This is taken into account in this model by means of the coefficient $f_{h}(h)$, also enters in the definition of the hydration degree [4], and which is given by

$$
f_{h}(h)=\frac{1}{1+\left(a_{h}-a_{h} h\right)^{b_{h}}}
$$

where the parameters $a_{h}$ and $b_{h}$ are assumed constant and equal to 8 and 4, respectively, from a best fitting of the experimental data [5].

The effect of the initial value of the crack width is reproduced by the coefficient $f_{w}\left(w_{c}\right)$ which has the following expression

$$
f_{w}\left(w_{c}\right)=\left\{1+\left[a_{w}-a_{w}\left(1-w_{c}\right)\right]^{b_{w}}\right\}^{-1}(19)
$$

with the addition that $f_{w}\left(w_{c}\right)=0$ as far as $w_{c}<w_{c 0}$. The coefficients $a_{w}$ and $b_{w}$ are two free parameter.

\subsection{Numerical implementation}

Since diffusion and mechanical problems must be coupled, in order to actually simulate the effect of cracks healing on moisture transport and heat transfer phenomena, and assess the strength recovery due to autogenous healing, such a process is implemented in both models presented in the previous sections.
Concerning the HTC model, the autogenous healing is introduced into the numerical code, paying attention to the condition of process activation: no self-healing can have place if threshold value, in terms of crack width, $w_{c 0}$, is not reached. As soon as $w_{c 0}$ occurs, the selfhealing module starts working and updating the solution, going through the calculation of the amount of unhydrated cement, which heavily influences the reaction affinity, thus the starting rate of the process.

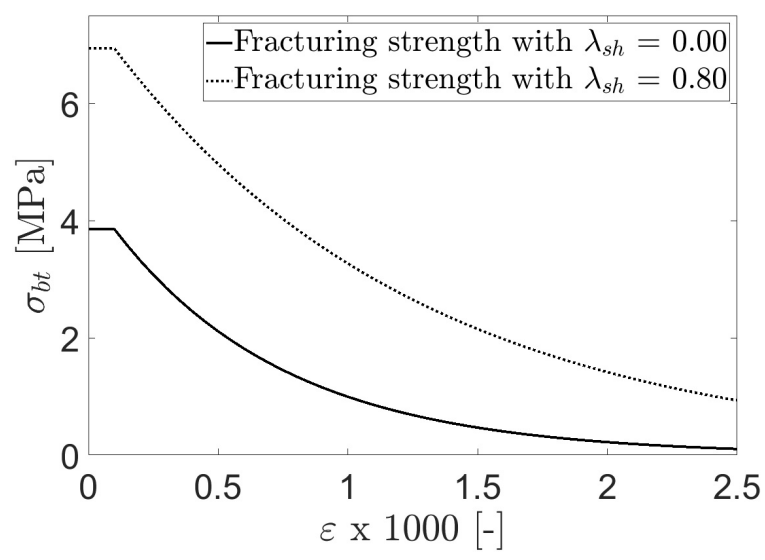

Figure 2: Homothetic expansion of the boundary curve representing the fracturing behaviour of the material (self-healing degree assumed equal to $80 \%$ ).

Going to look at LDPM, the self-healing effect must come into play when, once damage has occurred, the healing reaction permits to experience a recovery in strength. At this point, two ways seem reasonable and feasible, both acting on the constitutive law which governs the fracturing and cohesive behaviour under tension and tension/shear, here equation (12): 1) to simulate the recovery in tensile strength as a consequence of a recovery in normal strain; 2) to keep the normal strain constant after the healing and make the associated tensile strength grow proportionally to the actual self-healing degree. In this study the latter has been the modelling strategy investigated, implemented as showed in (20) and resulting in a homothetic expansion of the boundary curve (see Fig. 2).

$$
\sigma_{0}=\sigma_{0}\left(1+k_{s h} \lambda_{s h}\right)
$$


Once self-healing is implemented, LDPM and HTC advance towards the solution being twoway coupled. The two-way coupler, basically, makes the two algorithms communicate, although they have been conceived to advance simultaneously.

For the seek of clarity, it is worth underlining that the mechanical analysis usually performed are on the time scale of micro or mini seconds. On the other hand, a diffusion analysis, such as curing or ageing, is usually on the time scale of days, months or even years. Therefore, the time step for a diffusion analysis would be several orders of magnitude bigger than for the mechanical analysis. In a coupled analysis, if the same time step is adopted for both mechanical and diffusion analysis, the former will experience instability. To overcome this issue, a socalled time map is introduced into the code. It is used to scale down the time scale of a diffusion analysis (real time) such that the mechanical analysis (simulation time) has a reasonably small time step.

Going back to the coupled problem of interest, the process flow follows nine steps:

1. At $t=0$ the diffusion analysis advances by two time steps (one if $t>0$ ) and computes the solutions $f_{B}\left(t_{B}\right)$ and $f_{E}\left(t_{E}\right)$ (if $t>0, f_{B}$ and $f_{E}$ are however the two latest solutions);

2. the simulation time in the mechanical analysis is incremented by one time step and, by means of the time map, the real current time, $R C T$ is computed;

3. if $R C T>t_{E}$, it is necessary to go back to step (1), otherwise $f_{B}$ and $f_{E}$ are interpolated in time in order to determine the $f_{C}(R C T)$;

4. $f_{C}$ is interpolated in space in order to compute relative humidity and temperature on each LDPM facet;

5. LDPM modules (e.g. thermal strain module) takes the values at LDPM facets and computes strains and stresses;
6. LDPM increments the strain on each facet;

7. the mechanical analysis advances by one time step and computes the solution (stresses) at current time ( $C T$ which corresponds to $R C T$ in the simulation time);

8. the HTC takes information from LDPM to update its own solution;

9. when the convergence holds, it is possible to go back to step (1) and go ahead towards the final solution.

\section{EXPERIMENTAL CAMPAIGN}

The experimental campaign taken here as a reference for the numerical modelling has been described in detail in [21]. A Normal Strength Concrete, with a target average compressive strength of $30 \mathrm{~N} / \mathrm{mm}^{2}$ (on cubes) and a watercement ratio equal to 0.55 was taken as a reference, also addressing the influence of a crystalline admixture, employed as self healing promoter at a dosage equal to $1 \%$ by weight of cement, but not relevant to this study since it has not been included into the numerical simulation in object. However, for details about the chemical composition of the employed admixture, the results of SEM observations and the characterization of the healing products the reader is referred to [21].

The self-healing evaluation methodology employed un-notched prismatic beam specimens (500 mm x $100 \mathrm{~mm} \times 50 \mathrm{~mm})$ pre-cracked in three-point bending test up to two different crack opening levels, respectively equal to 0.15 and $0.30 \mathrm{~mm}$, and the either stored in water or left exposed to open air up to 12 months. Three-point bending tests were repeated up to failure after the scheduled conditioning periods. Self-healing was thus evaluated by comparing the peak-load bearing capacity exhibited by the specimens after conditioning to the residual one the same specimens featured upon pre-cracking when unloaded at the prescribed crack opening. 


\section{PARAMETERS CALIBRATION}

The calibration phase has been performed with respect to both mechanical and diffusion model; in particular, LDPM parameters have been calibrated in order to achieve a twofold aim: (1) to catch the peak in compressive strength experienced by specimens during unconfined compression test after a 28-day curing; (2) to simulate the fracturing behaviour which the specimens exhibited during pre-cracking three-point bending tests. Concerning the diffusion parameters, the 31-day curing before specimens pre-cracking has been simulated.

As first step towards numerical models calibration, the mesh generation settings within the numerical environment have been identified in order to achieve the best fitting possible between the experimental and the numerical sieve curves. This operation, obviously, has been performed paying attention to the coarse aggregates in the mixture in order to avoid a too refined mesh which would have resulted into high computational costs. As discussed in [7], the issue concerning the minimum aggregate size is relevant and hard to overcome, but it might be a good point to have a mesh able to simulate at least the $30 \%$ of the total aggregate particles. In this study the simulated aggregate volume is roughly equal to $32 \%$ and the matching between experimental and numerical curves seems to be quite good (see Fig. 4).

\subsection{Mechanical problem parameters}

The LDPM parameters have been defined by means of a best fitting between experimental and numerical results. In terms of compressive strength, LDPM catches the average peak which specimens exhibited in unconfined compression test after 28-day curing. As it is possible to see by looking at Figs. 6, LDPM is also capable of simulating the fracturing and softening behaviour of specimens quite precisely. The parameters which allow for this matching are $E_{0}=43000$ $\mathrm{MPa}, \alpha=0.25, \sigma_{t}=2.40 \mathrm{MPa}, \sigma_{c 0}=120 \mathrm{MPa}$, $\sigma_{s} / \sigma_{t}=3.50, l_{t}=180 \mathrm{~mm}, n_{t}=1.0, H_{c 0} / E_{0}=0.40$, $k_{c 0}=2.0, \quad k_{c 1}=1.0, \quad k_{c 2}=5.0, \quad \mu_{0}=0.2, \quad \mu_{\infty}=0.0$,
$\sigma_{N 0}=600 \mathrm{MPa}, \beta=0.0, k_{t}=0.5, k_{c}=0.5$, and $k_{s}=0.5$. Some of the parameters listed above might have not been presented within this paper because they mainly concern the other two LDPM response models for concrete: the pore collapse and material compaction, and frictional behaviour.

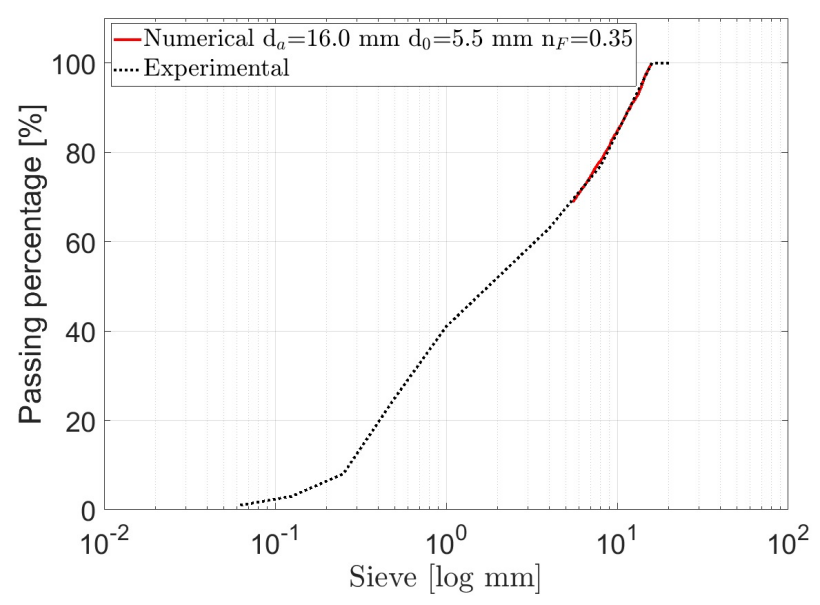

Figure 3: Comparison between numerical and experimental sieve curves.

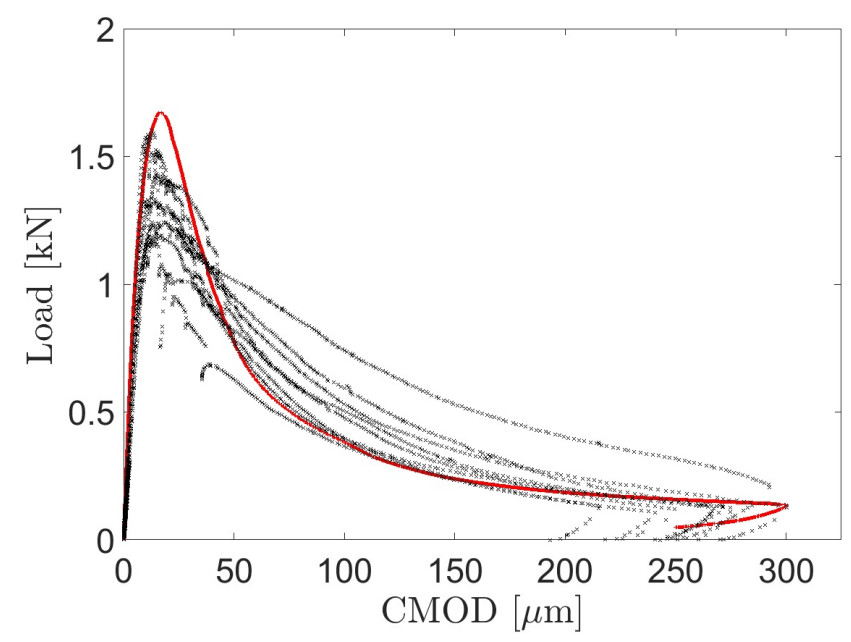

Figure 4: Comparison between numerical (red line) and experimental results (black crosses) of three-point bending tests carried out to precrack the specimens.

\subsection{Diffusion problem parameters}

The procedure for the calibration of the parameter of the Hygro-Thermo-Chemical (HTC) 
model was discussed minutely in [5]. Because the experimental data necessary to calibrate the HTC parameters are not available with reference to the concrete herein investigated [21], an educated guess of the parameter values is therefore necessary. In the presented study the following HTC parameters were used based on the previous experience on model calibration: $\rho=2400 \mathrm{~kg} / \mathrm{m}^{3} ; c_{t}=1100 \mathrm{~J} / \mathrm{kg}^{\circ} \mathrm{C} ; \lambda_{t}=5.4$ $\mathrm{W} / \mathrm{m}^{\circ} \mathrm{C} ; \tilde{Q}_{c}^{\infty}=500 \mathrm{~kJ} / \mathrm{kg} ; \tilde{Q}_{s}^{\infty}=780 \mathrm{~kJ} / \mathrm{kg}$; $E_{a c} / R=5000 \mathrm{~K} ; E_{a s} / R=9700 \mathrm{~K} ; E_{a d} / R=$ $2700 \mathrm{~K} ; S F^{e f f}=0.9 ; \kappa_{c}=0.253 ; g_{1}=1.3$; $k_{v g}^{c}=0.2 ; k_{v g}^{s}=0.3 ; A_{c 1}=3 \cdot 10^{7} \mathrm{~h}^{-1} ; A_{c 2}=$ $1 \cdot 10^{-4} ; \eta_{c}=5.5 ; A_{s 1}=20 \cdot 10^{11} \mathrm{~h}^{-1} ; A_{s 2}=$ $1 \cdot 10^{-6} ; \eta_{s}=9.5 ; D_{0}=1.2 \cdot 10^{-9}[\mathrm{~kg} / \mathrm{mm} \mathrm{h}]$; $D_{1}=8.3 \cdot 10^{-7}[\mathrm{~kg} / \mathrm{mm} \mathrm{h}] ; n=4.5$. The results refer to exposure conditions with $\mathrm{RH}=90 \%$ and $\mathrm{T}=20^{\circ} \mathrm{C}$ and, in terms of relative humidity field (see Fig. 7), seem to be reasonable, whereas the temperature within the specimen keeps constant over the time.

The new parameters of the self-healing recovery degree (see Eqs. 13, 17) are still a guess since the healing modelling is under validation. The following values have been set, accordingly to [3]: $\widetilde{A}_{s h 1}=3 \cdot 10^{3} \mathrm{~m}^{3} / \mathrm{kg} / \mathrm{h}, \widetilde{A}_{s h 2}=3.25 \cdot 10^{4}$ $\mathrm{m}^{3} / \mathrm{kg} / \mathrm{h}, a_{w}=3, b_{w}=4$, and $E_{s h} / R=5000 \mathrm{~K}$.

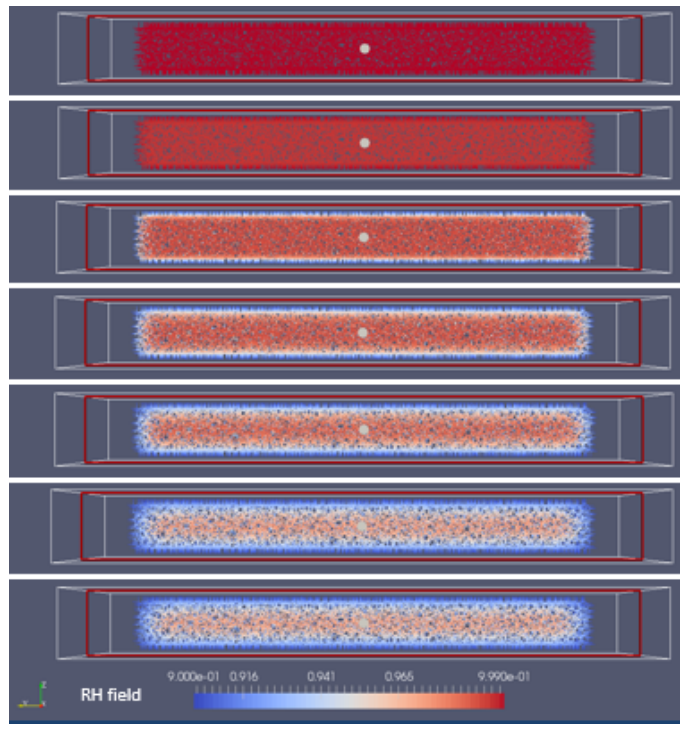

Figure 5: Relative humidity field at mid longitudinal section of the specimen at 1, 3, 6, 12, 28 and 31 days

\section{NUMERICAL RESULTS}

Aiming at simulating the self-healinginduced recovery in strength experienced by the material after a 12-month exposure period, an input file for a coupled diffusion and mechanical analysis has been prepared. In particular, the simulation is set to consist of: (1) 1 month curing at $\mathrm{RH}=90 \%$ and $\mathrm{T}=20^{\circ} \mathrm{C}$; (2) $3 \mathrm{PBT}$ up to a residual CMOD of $240 \mu \mathrm{m}$; (3) 12-month exposure whether in water or open air with $\mathrm{RH}=90 \%$ and $\mathrm{T}=20^{\circ} \mathrm{C}$; (4) 3PBT up to failure. This study has dealt with the simulations of specimens immersed in water, once pre-cracked.

Looking at Fig. 8 what stands out is the evidence that no autogenous healing has been experienced by the material, thus no recovery in strength is visible on the graph.

\section{CONCLUSIONS}

Some conclusive remarks are presented here. - LDPM is capable of simulating the mechanical response of the material whether in terms of compressive strength and regarding fracturing strength; concerning the latter, it has proved that numerical results are totally overlapping to the scatter of experimental results achieved by carrying out three-point bending tests.

- To improve the quality of models calibration more than one mesh need to be simulated, varying the starting seed for the random process of particles positioning. This would make the results more reliable.

- HTC gives quite reasonable results in terms of relative humidity field, whereas the temperature holds constant over all the 31-day curing. It might be due to the extremely low thickness of the specimen (only $50 \mathrm{~mm}$ ), which leads to the hypothesise that the thermal equilibrium is quickly achieved whenever internal exothermal reactions induce an increment in temperature.

- The implementation of self-healing leads to a sub-homothetic expansion of the boundary curve relevant to the fracturing behaviour.

- It is evident that self-healing does not perform properly with the current set of simulation configuration: probably it would be needed to keep taking action on the parameters in order to ob- 
tain not zero results. Apparently, the process does not even start. This is the most relevant current issue.

The work described in this paper has been performed in the framework of the project ReSHEALience - Rethinking coastal defence and Green-energy Service infrastructures through enHancEd-durAbiLity highperformance cement-based materials (WP6). This project has received funding from the European Union's Horizon 2020 research and innovation programme under grant agreement No 760824.

The information and views set out in this publication do not necessarily reflect the official opinion of the European Commission. Neither the European Union institutions and bodies nor any person acting on their behalf, may be held responsible for the use which may be made of the information contained therein.

The numerical analyses have been performed by means of Mars-UD, an explicit dynamic code distributed by ES3 Inc. (Engineering and Software System Solutions).

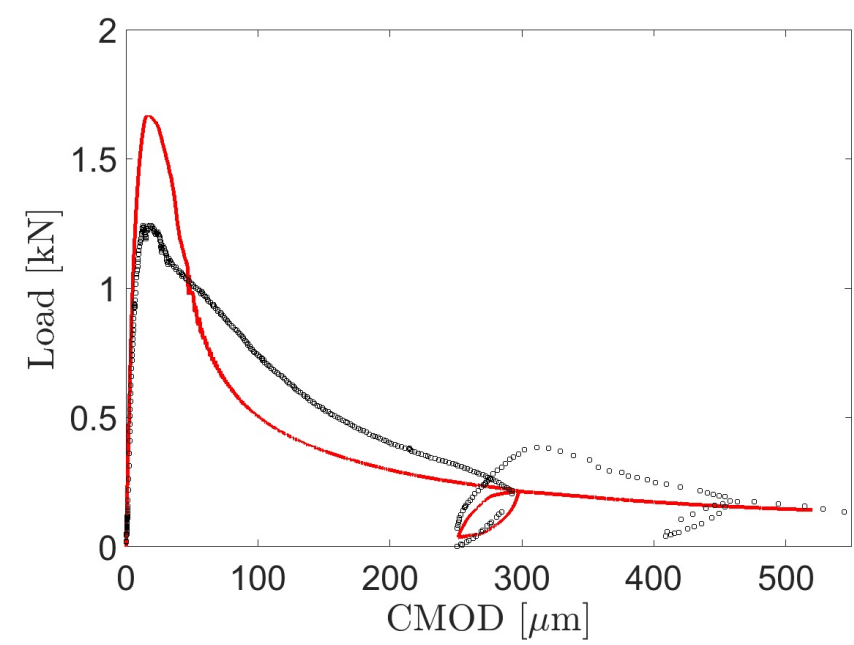

Figure 6: Comparison between numerical (red line) and experimental results (black crosses) of three-point bending tests carried out to precrack the specimens.

\section{REFERENCES}

[1] Di Luzio, G., Ferrara, L., Alonso, M.C., Kunz, P., Mechtcherine, V., Schroefl, C.
2018. Predicting the long-term performance of structures made with advanced cement based materials in extremely aggressive environments: current state of practice and research needs. The approach of H2020 project ReSHEALience. Symposium on Concrete Modelling ConMOD2018; pp. 467-477.

[2] Di Luzio, G., and Cusatis, G. 2013. Solidification-Microprestress-Microplane (SMM) theory for concrete at early age: theory, validation and application. International Journal of Solids and Structures 50 (2009b); pp. 957-975.

[3] Di Luzio, G., Ferrara, L., Krelani, V. 2017. Numerical modeling of mechanical regain due to self-healing in cement based composites. Cement and Concrete Composites 86; pp. 190-205.

[4] Di Luzio, G., and Cusatis, G. 2009. Hygro-thermo-chemical modeling of high performance concrete. I: Theory. Cement and Concrete Composites 58 (2009a); pp. 301-308.

[5] Di Luzio, G., and Cusatis, G. 2009. Hygro-thermo-chemical modeling of high performance concrete. II: Numerical implementation, calibration, and validation. Cement and Concrete Composites $\mathbf{5 8}$ (2009a); pp. 309-324.

[6] Cusatis, G., Pelessone, D., Mencarelli, A. 2011. Lattice Discrete Particle Model (LDPM) for failure behavior of concrete. I: Theory. Cement and Concrete Composites 33; pp. 881-890.

[7] Cusatis, G., Mencarelli, A., Pelessone, D., Baylot, J. 2011. Lattice Discrete Particle Model (LDPM) for failure behavior of concrete. II: Calibration and validation. Cement and Concrete Composites 33; pp. 891-905.

[8] Taylor, H. 1997. Cement chemistry. Thomas Telford London. 
[9] Neville, A. 1997. Properties of concrete. John Wiley and Sons New York, USA.

[10] Ulm, F.J., and Coussy, O. 1995. Modeling of thermochemomechanical couplings of concrete at early ages. Journal of Engineering Mechanics ASCE 121; pp. 785794.

[11] Cervera, M., Oliver, J., Prato, T. 1999. Thermo-chemo-mechanical model for concrete. I: hydration and ageing. Journal of Engineering Mechanics ASCE 125; pp. 1018-1027.

[12] Gawin, D., Pesavento, F., Schrefler, B.A. 2006. Hygro-thermo-chemo-mechanical modelling of concrete at early ages and beyond. part i: hydration and hygro-thermal phenomena. International Journal for Numerical Methods in Engineering 67; pp. 299-331.

[13] Pantazopoulo, S., and Mills, R. 1995. Microstructural aspects of the mechanical response of plain concrete. ACI Materials Journal 92; pp. 605-616.

[14] Duchesne, J., and Bérubé, M.A. 1994. The effectiveness of supplementary cementing materials in suppressing expansion due to ASR: another look at the reaction mechanisms Part 1: concrete expansion and portlandite depletion. Cement and Concrete Research 24(1); pp. 73-82.

[15] Persson, B. 1998. Seven-year study on the effect of silica fume in concrete. Advanced Cement Based Materials 7; pp. 139-155.

[16] Ferrara, L., Van Mullen, T., Alonso, M.C., Antonaci, P., Borg, R.P., Cuenca, E., Jefferson, A., Ng, P.L., Peled, A., RoigFlores, M., Sanchez, M., Schroefl, C., Serna, P., Snoeck, D., Tulliani, J.M., De Belie, N., 2018. Experimental characterization of the self-healing capacity of cement based materials and its effects on the material performance: A state of the art report by COST Action SARCOS WG2. Construction and Building Materials. 167, pp. 115-142.

[17] Atkins, P. W. (1994). Physical chemistry (5th Ed.). Oxford University Press Oxford, U.K.

[18] Edvardsen, C. 1996. Water permeability and self-healing of through-crack in concrete. (in German). DAfStb Bull. 455, Berlin.

[19] TC RILEM 221-SHC. 2013. Self-Healing Phenomena in Cement-Based Materials State-of-the-Art Report of RILEM Technical Committee 221-SHC: Self-Healing Phenomena in Cement-Based Materials. Volume RILEM State-of-the-Art Reports. Springer.

[20] Ferrara, L., Krelani, V., Moretti, F. 2016. Autogenous healing on the recovery of mechanical performance of High Performance Fibre Reinforced Cementitious Composites (HPFRCCs): Part 2 - Correlation between healing of mechanical performance and crack sealing. Cement and Concrete Composites 73; pp. 299-315.

[21] Ferrara, L., Krelani, V., Carsana, M. 2014. A "fracturing test" based approach to assess crack healing of concrete with and without crystalline admixtures. Construction and Building Materials. 68, pp. 535551.

[22] Jefferson, T., Javierre, E., Lee Freeman, B., Zaoui, A., Koenders, E., Ferrara, L. 2018. Research progress on numerical models for self-healing cementitious materials. Advanced Materials and Interfaces 5(17); pp. 1-19. 